International Journal of Pure and Applied Mathematics

Volume 97 No. 4 2014, 409-418

ISSN: 1311-8080 (printed version); ISSN: 1314-3395 (on-line version)

url: http://www.ijpam.eu

doi: http://dx.doi.org/10.12732/ijpam.v97i4.3

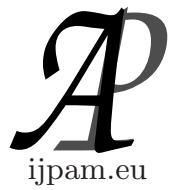

\title{
QUATERNIONIC COUPLING
}

\author{
J. Marão ${ }^{1}$, M.F. Borges ${ }^{2} \S$ \\ ${ }^{1}$ UFMA - Federal University of Maranhão \\ B.Sc. Interdisciplinary Science and Technology - (BCT) \\ 65085-580, Maranhão, BRAZIL \\ ${ }^{2}$ UNESP - São Paulo State University \\ S.J. Rio Preto Campus \\ 15054-000, São José do Rio Preto, BRAZIL
}

\begin{abstract}
Physics is in its development a major challenge to relate fields, this paper presents a proposal to relate classical fields of physics, ie the electric field, magnetic field and gravitational equations by time-dependent. The proposal begins with the work that determines the Cauchy-Riemann conditions for quaternions [1], and the determination of Laplace's equation in four dimensions[3], it was possible to determine mathematical components important to make the couplings of classical fields discussed above.
\end{abstract}

AMS Subject Classification: 30G99, 30E99

Key Words: quaternions, Laplace's equations, geoemtrical coupling fields

\section{Introduction}

Initially will present the classical equations for the gravitational field and the magnetic field, these equations that take the form of Laplace's equations by means of transformations that will be shown at convenient time.

The following equation is known as Gauss's law for gravity, and it is clear that the potential function that makes the equation are functions of the vari-

Received: December 23, 2013

(c) 2014 Academic Publications, Ltd.

$\S_{\text {Correspondence author }}$ url: www.acadpubl.eu 
ables $t, x, y$ and $z .:$

$$
\nabla^{2} \phi(t, x, y, z)=4 \pi G \rho(t, x, y, z)
$$

The law of gravitation to Gauss: $\phi(t, x, y, z)$ is the gravitational potential $G$ a gravitational constant and $\rho(t, x, y, z)$ the density.

One others classical equations mentioned will be important for our scope of searching for a coupling theory in a hypercomplex and involving gravitational, electrical and magnetic fields

$$
\begin{aligned}
-\frac{1}{c^{2}} \frac{\partial^{2} \mathbf{A}}{\partial t^{2}}(t, x, y, z)+\frac{\partial^{2} \mathbf{A}}{\partial x^{2}}(t, x, y, z)+\frac{\partial^{2} \mathbf{A}}{\partial y^{2}}(t, x, y, z)+ & \frac{\partial^{2} \mathbf{A}}{\partial z^{2}}(t, x, y, z) \\
& =-\frac{4 \pi}{c} \mathbf{J}(t, x, y, z),
\end{aligned}
$$

which can be written as the following set of equations:

$$
\begin{array}{r}
-\frac{1}{c^{2}} \frac{\partial^{2} A_{x}}{\partial t^{2}}(t, x, y, z)+\frac{\partial^{2} A_{x}}{\partial x^{2}}(t, x, y, z)+\frac{\partial^{2} A_{x}}{\partial y^{2}}(t, x, y, z)+\frac{\partial^{2} A_{x}}{\partial z^{2}}(t, x, y, z) \\
=-\frac{4 \pi}{c} J_{x}(t, x, y, z), \\
-\frac{1}{c^{2}} \frac{\partial^{2} A_{y}}{\partial t^{2}}(t, x, y, z)+\frac{\partial^{2} A_{y}}{\partial x^{2}}(t, x, y, z)+\frac{\partial^{2} A_{y}}{\partial y^{2}}(t, x, y, z)+\frac{\partial^{2} A_{y}}{\partial z^{2}}(t, x, y, z) \\
=-\frac{4 \pi}{c} J_{y}(t, x, y, z),
\end{array}
$$

and

$$
\begin{array}{r}
-\frac{1}{c^{2}} \frac{\partial^{2} A_{z}}{\partial t^{2}}(t, x, y, z)+\frac{\partial^{2} A_{z}}{\partial x^{2}}(t, x, y, z)+\frac{\partial^{2} A_{z}}{\partial y^{2}}(t, x, y, z)+\frac{\partial^{2} A_{z}}{\partial z^{2}}(t, x, y, z) \\
=-\frac{4 \pi}{c} J_{z}(t, x, y, z),
\end{array}
$$

where $\mathbf{A}=\left(A_{x}, A_{y}, A_{z}\right)$ is the magnetic potential and $\mathbf{J}=\left(J_{x}, J_{y}, J_{z}\right)$ is regarded as the current density.

The above equations, after appropriate processing, allows it to be studied as the Laplace's equations, as seen in [3]:

$$
\sum_{i=1}^{4} \frac{\partial^{2} F}{\partial x_{i}^{2}}=0, \quad i=1,2,3,4,
$$

where $F$ is real function of variables $t, x, y, z$. 


\section{Obtaining the Laplace's Equations}

Let us considering $f(q)$ where $q$ is an quaternionic number and $f(q)$ is called quaternionic function. We can associate with it a set of equations called the Cauchy-Riemann like relations [1]. Firstly, will be presented without proof, a theorem of important Classical Complex Analysis and guide the development of this work. Consider

Theorem 1. If $f(z)=u(x, y)+i v(x, y)$ a function defined and continues in a neighborhood of the point $z=x+i y$ and differenciable in $z$. Then the partial derivatives of the first order of $u(x, y)$ and $v(x, y)$ exist and satisfy the relations:

$$
\frac{\partial u}{\partial x}(x, y)=\frac{\partial v}{\partial y}(x, y)
$$

and

$$
\frac{\partial u}{\partial y}(x, y)=-\frac{\partial v}{\partial x}(x, y)
$$

The set of equations (5) and (6) are called Cauchy-Riemann relations [4]. If the set of functions above is class of $C^{2}$, then they satisfy the Schwartz's theorem, then it follows that:

$$
\nabla^{2} u(x, y)=0
$$

and

$$
\nabla^{2} v(x, y)=0 .
$$

The results obtained in [3] and [1] can be analyzed within physical focus by making the following change of variables:

$$
\begin{aligned}
x_{1} & =t \\
x_{2} & =x \\
x_{3} & =y \\
x_{4} & =z
\end{aligned}
$$

Theorem 2. For any pair pontis $a$ and $b$ and any path joining them simply conect subdomain of the four-dimmensional space, the integral $\int_{a}^{b} f d q$ is independent form the given path if and only if there is a function $F=$ $F_{1}+F_{2} i+F_{3} j+F_{4} k$ such that $\int_{a}^{b} f d q=F(a)-F(b)$, and satisfying the following relations:

$$
\frac{\partial F_{1}}{\partial t}=\frac{\partial F_{2}}{\partial x}=\frac{\partial F_{3}}{\partial y}=\frac{\partial F_{4}}{\partial z}
$$




$$
\begin{gathered}
\frac{\partial F_{2}}{\partial t}=-\frac{\partial F_{1}}{\partial x}=-\frac{\partial F_{3}}{\partial z}=\frac{\partial F_{4}}{\partial y} \\
\frac{\partial F_{3}}{\partial t}=-\frac{\partial F_{1}}{\partial y}=-\frac{\partial F_{2}}{\partial z}=\frac{\partial F_{4}}{\partial x} \\
\frac{\partial F_{4}}{\partial t}=\frac{\partial F_{1}}{\partial z}=-\frac{\partial F_{2}}{\partial y}=-\frac{\partial F_{3}}{\partial x}
\end{gathered}
$$

Proof. The proof of this theorem can be analyzed in greater detail in [1].

Now making the derivation of the above relations, admitting that the real functions in question are of class $C^{2}$. The followings equations are obtained [3]:

$$
\begin{aligned}
& \frac{\partial^{2} F_{1}}{\partial^{2} t^{2}}+\frac{\partial^{2} F_{1}}{\partial x^{2}}+\frac{\partial^{2} F_{1}}{\partial y^{2}}+\frac{\partial^{2} F_{1}}{\partial z^{2}}=0 \\
& \frac{\partial^{2} F_{2}}{\partial t^{2}}+\frac{\partial^{2} F_{2}}{\partial x^{2}}+\frac{\partial^{2} F_{2}}{\partial y^{2}}+\frac{\partial^{2} F_{2}}{\partial z^{2}}=0 \\
& \frac{\partial^{2} F_{3}}{\partial t^{2}}+\frac{\partial^{2} F_{3}}{\partial x^{2}}+\frac{\partial^{2} F_{3}}{\partial y^{2}}+\frac{\partial^{2} F_{3}}{\partial z^{2}}=0
\end{aligned}
$$

and

$$
\frac{\partial^{2} F_{4}}{\partial t^{2}}+\frac{\partial^{2} F_{4}}{\partial x^{2}}+\frac{\partial^{2} F_{4}}{\partial y^{2}}+\frac{\partial^{2} F_{4}}{\partial z^{2}}=0
$$

The result shows how it behaves to the functions of the complex components, a change of variable of type time translation. Consider:

Theorem 3. If $G$ is an function of $t, x, y$ e $z$, variables, then the translation of $t$ for $t^{\prime}$, of temporal coordinates, satisfies the following equation:

$$
\frac{\partial^{2} G}{\partial t^{2}}(t, x, y, z)=\frac{\partial^{2} G^{\prime}}{\partial t^{\prime 2}}\left(t^{\prime}, x, y, z\right)
$$

where $G^{\prime}$ is the $G$ applied at time $t^{\prime}$ for the same spatial coordinates $x, y$ e $z$.

Proof. Initially:

$$
\frac{\partial G}{\partial t}(t, x, y, z)=\frac{\partial G}{\partial t^{\prime}}\left(t^{\prime}, x, y, z\right) \frac{d t^{\prime}}{d t}=\frac{\partial G}{\partial t^{\prime}}\left(t^{\prime}, x, y, z\right)
$$

The derivative is given for:

$$
\frac{\partial^{2} G}{\partial t^{2}}(t, x, y, z)=\frac{\partial^{2} G^{\prime}}{\partial t^{\prime} 2}\left(t^{\prime}, x, y, z\right) \frac{d t^{\prime}}{d t}=\frac{\partial^{2} G^{\prime}}{\partial t^{\prime} 2}\left(t^{\prime}, x, y, z\right)
$$


The results below can also be seen in [5].

Note. For mixed derivatives with respect to time and spatial coordinates $x$, $y$ or $z$, the translation is easily obtained according to the following calculation for a hypothetical situation:

$$
\begin{aligned}
\frac{\partial^{2} G}{\partial t \partial x}(t, x, y, z)=\frac{\partial}{\partial t}\left(\frac{\partial G}{\partial x}\right)(t, x, y, z)=\frac{\partial}{\partial t^{\prime}}\left(\frac{\partial G^{\prime}}{\partial x}\right)\left(t^{\prime}, x, y, z\right) \frac{d t^{\prime}}{d t} & \\
& =\frac{\partial^{2} G^{\prime}}{\partial t^{\prime} \partial x}\left(t^{\prime}, x, y, z\right)
\end{aligned}
$$

Seeking to adapt the equations given in (1), (2) and (3) to Laplace's equation obtained earlier, it will be used the following change in the configuration of derivatives against time. After translating the coordinates in second derivative of $f(t, x, y, z)$ relative to $t$ it is performed the second derivative of $F^{\prime}\left(t^{\prime}, x, y, z\right)$ relative to $t$ plus a function $\varphi\left(t^{\prime}, x, y, z\right)$ as follows:

$$
\frac{\partial^{2} F}{\partial t^{2}}(t, x, y, z)=\frac{\partial^{2} F^{\prime}}{\partial t^{2}}\left(t^{\prime}, x, y, z\right)+\varphi\left(t^{\prime}, x, y, z\right)
$$

\section{Transformations in Laplace's Equations}

Let us consider, $f(q)=F_{1}(t, x, y, z)+F_{2}(t, x, y, z) i+F_{3}(t, x, y, z) j+F_{4}(t, x, y, z) k$ an quaternionic function where $F_{i}(q) i=1,2,3,4$ are functions of real variables that satisfies the Cauchy-Riemann conditions.

Deriving the conditions of equation (9), (10), (11) and (12) we have that:

$$
\begin{aligned}
& \frac{\partial^{2} F_{1}}{\partial t^{2}}=\frac{\partial^{2} F_{2}}{\partial t \partial x}=\frac{\partial^{2} F_{3}}{\partial t \partial y}=\frac{\partial^{2} F_{4}}{\partial t \partial z} \\
& \frac{\partial^{2} F_{1}}{\partial t \partial x}=\frac{\partial^{2} F_{2}}{\partial x^{2}}=\frac{\partial^{2} F_{3}}{\partial x \partial y}=\frac{\partial^{2} F_{4}}{\partial x \partial z} \\
& \frac{\partial^{2} F_{1}}{\partial y \partial t}=\frac{\partial^{2} F_{2}}{\partial y \partial x}=\frac{\partial^{2} F_{3}}{\partial y^{2}}=\frac{\partial^{2} F_{4}}{\partial z \partial y} \\
& \frac{\partial^{2} F_{1}}{\partial t \partial z}=\frac{\partial^{2} F_{2}}{\partial z \partial x}=\frac{\partial^{2} F_{3}}{\partial z \partial y}=\frac{\partial^{2} F_{4}}{\partial z^{2}} .
\end{aligned}
$$




$$
\begin{aligned}
& \frac{\partial^{2} F_{2}}{\partial t^{2}}=-\frac{\partial^{2} F_{1}}{\partial t \partial x}=-\frac{\partial^{2} F_{3}}{\partial t \partial z}=\frac{\partial^{2} F_{4}}{\partial t \partial y} \\
& \frac{\partial^{2} F_{2}}{\partial t \partial x}=-\frac{\partial^{2} F_{1}}{\partial x^{2}}=-\frac{\partial^{2} F_{3}}{\partial x \partial z}=\frac{\partial^{2} F_{4}}{\partial y \partial x} \\
& \frac{\partial^{2} F_{2}}{\partial y \partial t}=-\frac{\partial^{2} F_{1}}{\partial y \partial x}=-\frac{\partial^{2} F_{3}}{\partial y \partial z}=\frac{\partial^{2} F_{4}}{\partial y^{2}} \\
& \frac{\partial^{2} F_{2}}{\partial z \partial t}=-\frac{\partial^{2} F_{1}}{\partial z \partial x}=-\frac{\partial^{2} F_{3}}{\partial z^{2}}=\frac{\partial^{2} F_{4}}{\partial z \partial y} . \\
& \frac{\partial^{2} F_{3}}{\partial t^{2}}=-\frac{\partial^{2} F_{1}}{\partial t \partial y}=-\frac{\partial^{2} F_{2}}{\partial t \partial z}=\frac{\partial^{2} F_{4}}{\partial t \partial x} \\
& \frac{\partial^{2} F_{3}}{\partial t \partial x}=-\frac{\partial^{2} F_{1}}{\partial x \partial y}=-\frac{\partial^{2} F_{2}}{\partial x \partial z}=\frac{\partial^{2} F_{4}}{\partial x^{2}} \\
& \frac{\partial^{2} F_{3}}{\partial y \partial t}=-\frac{\partial^{2} F_{1}}{\partial y^{2}}=-\frac{\partial^{2} F_{2}}{\partial z \partial y}=\frac{\partial^{2} F_{4}}{\partial y \partial x} \\
& \frac{\partial^{2} F_{3}}{\partial t \partial z}=-\frac{\partial^{2} F_{1}}{\partial z \partial y}=-\frac{\partial^{2} F_{2}}{\partial z^{2}}=\frac{\partial^{2} F_{4}}{\partial z \partial x} . \\
& \frac{\partial^{2} F_{4}}{\partial t^{2}}=\frac{\partial^{2} F_{1}}{\partial t \partial z}=-\frac{\partial^{2} F_{2}}{\partial t \partial y}=-\frac{\partial^{2} F_{3}}{\partial t \partial x} \\
& \frac{\partial^{2} F_{4}}{\partial t \partial x}=\frac{\partial^{2} F_{1}}{\partial x \partial z}=-\frac{\partial^{2} F_{2}}{\partial x \partial y}=-\frac{\partial^{2} F_{3}}{\partial x^{2}} \\
& \frac{\partial^{2} F_{4}}{\partial y \partial t}=\frac{\partial^{2} F_{1}}{\partial y \partial z}=-\frac{\partial^{2} F_{2}}{\partial y^{2}}=-\frac{\partial^{2} F_{3}}{\partial y \partial x} \\
& \frac{\partial^{2} F_{4}}{\partial t \partial z}=\frac{\partial^{2} F_{1}}{\partial z^{2}}=-\frac{\partial^{2} F_{2}}{\partial z \partial y}=-\frac{\partial^{2} F_{3}}{\partial z \partial x} .
\end{aligned}
$$

Laplace's equations obtained here have no physical meaning yet. Are now made the following identifications: $\phi(t, x, y, z)=F_{1}(t, x, y, z), F_{2}(t, x, y, z)=$ $A_{x}(t, x, y, z), F_{3}(t, x, y, z)=A_{y}(t, x, y, z)$ and $F_{4}(t, x, y, z)=A_{z}(t, x, y, z)$. Therefore, the equations may have physical significance using the principle of translation plus function $\varphi$, and this will be done on each of the equation (1) as follows:

$$
\frac{\partial F_{1}}{\partial t^{2}}+\frac{\partial F_{1}}{\partial x^{2}}+\frac{\partial F_{1}}{\partial y^{2}}+\frac{\partial F_{1}}{\partial z^{2}}=0
$$

Making in (23) the translation and associating the function $\varphi\left(t^{\prime}, x, y, z\right)$ to $4 \pi G \rho\left(t^{\prime}, x, y, z\right)$, it generates a new $F^{\prime}$ as follows:

$$
\begin{array}{r}
{\left[\frac{\partial^{2} F_{1}^{\prime}}{\partial t^{\prime 2}}\left(t^{\prime}, x, y, z\right)-4 \pi G \rho\left(t^{\prime}, x, y, z\right)\right]+\frac{\partial^{2} F_{1}^{\prime}}{\partial x^{2}}(t, x, y, z)+\frac{\partial^{2} F_{1}^{\prime}}{\partial y^{2}}(t, x, y, z)} \\
+\frac{\partial^{2} F_{1}^{\prime}}{\partial z^{2}}\left(t^{\prime}, x, y, z\right)=0,
\end{array}
$$


this equation represents a Gauss-like law for gravitation.

By proceeding in a similar manner to the equations (2), (3) and (4), follows:

$$
\begin{gathered}
{\left[-\frac{1}{c^{2}} \frac{\partial^{2} F_{2}^{\prime}}{\partial t^{\prime 2}}(t, x, y, z)+\frac{4 \pi}{c} J_{x}(t, x, y, z)\right]+\frac{\partial^{2} F_{2}^{\prime}}{\partial x^{2}}(t, x, y, z)+\frac{\partial^{2} F_{2}^{\prime}}{\partial y^{2}}(t, x, y, z)} \\
+\frac{\partial^{2} F_{2}^{\prime}}{\partial z^{2}}(t, x, y, z)=0, \\
{\left[-\frac{1}{c^{2}} \frac{\partial^{2} F_{3}^{\prime}}{\partial t^{\prime 2}}(t, x, y, z)+\frac{4 \pi}{c} J_{x}(t, x, y, z)\right]+\frac{\partial^{2} F_{3}^{\prime}}{\partial x^{2}}(t, x, y, z)+\frac{\partial^{2} F_{3}^{\prime}}{\partial y^{2}}(t, x, y, z)} \\
+\frac{\partial^{2} F_{3}^{\prime}}{\partial z^{2}}(t, x, y, z)=0,
\end{gathered}
$$

and

$$
\begin{array}{r}
{\left[-\frac{1}{c^{2}} \frac{\partial^{2} F_{4}^{\prime}}{\partial t^{\prime 2}}(t, x, y, z)+\frac{4 \pi}{c} J_{z}(t, x, y, z)\right]+\frac{\partial^{2} F_{4}^{\prime}}{\partial x^{2}}(t, x, y, z)+\frac{\partial^{2} F_{4}^{\prime}}{\partial y^{2}}(t, x, y, z)} \\
+\frac{\partial^{2} F_{4}^{\prime}}{\partial z^{2}}(t, x, y, z)=0, \quad(27)
\end{array}
$$

Comparing the equations (24), (25), (26) and (27) with the previous sets of equations (19), (20), (21) and (22), and taking into account the invariance of the derived translational $t$ para $t^{\prime}$, we obtain the following sets:

$$
\begin{gathered}
\frac{\partial^{2} F_{1}^{\prime}}{\partial t^{\prime 2}}\left(t^{\prime}, x, y, z\right)-4 \pi G \rho\left(t^{\prime}, x, y, z\right)=\frac{\partial^{2} F_{2}^{\prime}}{\partial t^{\prime} \partial x}=\frac{\partial^{2} F_{3}^{\prime}}{\partial t^{\prime} \partial y}=\frac{\partial^{2} F_{4}^{\prime}}{\partial t^{\prime} \partial z} \\
\frac{\partial^{2} F_{1}^{\prime}}{\partial t^{\prime} \partial x}=\frac{\partial^{2} F_{2}^{\prime}}{\partial x^{2}}=\frac{\partial^{2} F_{3}^{\prime}}{\partial x \partial y}=\frac{\partial^{2} F_{4}^{\prime}}{\partial x \partial z} \\
\frac{\partial^{2} F_{1}^{\prime}}{\partial y \partial t^{\prime}}=\frac{\partial^{2} F_{2}^{\prime}}{\partial y \partial x}=\frac{\partial^{2} F_{3}}{\partial y^{2}}=\frac{\partial^{2} F_{4}^{\prime}}{\partial z \partial y} \\
\frac{\partial^{2} F_{1}^{\prime}}{\partial t^{\prime} \partial z}=\frac{\partial^{2} F_{2}^{\prime}}{\partial z \partial x}=\frac{\partial^{2} F_{3}^{\prime}}{\partial z \partial y}=\frac{\partial^{2} F_{4}}{\partial z^{2}} . \\
-\frac{1}{c^{2}} \frac{\partial^{2} F_{2}^{\prime}}{\partial t^{\prime 2}}(t, x, y, z)+\frac{4 \pi}{c} J_{x}(t, x, y, z)=-\frac{\partial^{2} F_{1}^{\prime}}{\partial t^{\prime} \partial x}=-\frac{\partial^{2} F_{3}^{\prime}}{\partial t^{\prime} \partial z}=\frac{\partial^{2} F_{4}^{\prime}}{\partial t^{\prime} \partial y} \\
\frac{\partial^{2} F_{2}^{\prime}}{\partial t^{\prime} \partial x}=-\frac{\partial^{2} F_{1}^{\prime}}{\partial x^{2}}=-\frac{\partial^{2} F_{3}^{\prime}}{\partial x \partial z}=\frac{\partial^{2} F_{4}^{\prime}}{\partial y \partial x} \\
\frac{\partial^{2} F_{2}^{\prime}}{\partial y \partial t^{\prime}}=-\frac{\partial^{2} F_{1}^{\prime}}{\partial y \partial x}=-\frac{\partial^{2} F_{3}^{\prime}}{\partial y \partial z}=\frac{\partial^{2} F_{4}^{\prime}}{\partial y^{2}} \\
\frac{\partial^{2} F_{2}^{\prime}}{\partial z \partial t^{\prime}}=-\frac{\partial^{2} F_{1}^{\prime}}{\partial z \partial x}=-\frac{\partial^{2} F_{3}^{\prime}}{\partial z^{2}}=\frac{\partial^{2} F_{4}^{\prime}}{\partial z \partial y} .
\end{gathered}
$$




$$
\begin{gathered}
-\frac{1}{c^{2}} \frac{\partial^{2} F_{3}^{\prime}}{\partial t^{\prime 2}}(t, x, y, z)+\frac{4 \pi}{c} J_{y}(t, x, y, z)=-\frac{\partial^{2} F_{1}^{\prime}}{\partial t^{\prime} \partial y}=-\frac{\partial^{2} F_{2}^{\prime}}{\partial t^{\prime} \partial z}=\frac{\partial^{2} F_{4}^{\prime}}{\partial t^{\prime} \partial x} \\
\frac{\partial^{2} F_{3}^{\prime}}{\partial t^{\prime} \partial x}=-\frac{\partial^{2} F_{1}^{\prime}}{\partial x \partial y}=-\frac{\partial^{2} F_{2}^{\prime}}{\partial x \partial z}=\frac{\partial^{2} F_{4}^{\prime}}{\partial x^{2}} \\
\frac{\partial^{2} F_{3}^{\prime}}{\partial y \partial t^{\prime}}=-\frac{\partial^{2} F_{1}}{\partial y^{2}}=-\frac{\partial^{2} F_{2}^{\prime}}{\partial z \partial y}=\frac{\partial^{2} F_{4}^{\prime}}{\partial y \partial x} \\
\frac{\partial^{2} F_{3}^{\prime}}{\partial t^{\prime} \partial z}=-\frac{\partial^{2} F_{1}^{\prime}}{\partial z \partial y}=-\frac{\partial^{2} F_{2}}{\partial z^{2}}=\frac{\partial^{2} F_{4}^{\prime}}{\partial z \partial x} . \\
-\frac{1}{c^{2}} \frac{\partial^{2} F_{4}^{\prime}}{\partial t^{\prime 2}}(t, x, y, z)+\frac{4 \pi}{c} J_{z}(t, x, y, z)=\frac{\partial^{2} F_{1}^{\prime}}{\partial t^{\prime} \partial z}=-\frac{\partial^{2} F_{2}^{\prime}}{\partial t^{\prime} \partial y}=-\frac{\partial^{2} F_{3}^{\prime}}{\partial t^{\prime} \partial x} \\
\frac{\partial^{2} F_{4}^{\prime}}{\partial t^{\prime} \partial x}=\frac{\partial^{2} F_{1}^{\prime}}{\partial x \partial z}=-\frac{\partial^{2} F_{2}^{\prime}}{\partial x \partial y}=-\frac{\partial^{2} F_{3}^{\prime}}{\partial x^{2}} \\
\frac{\partial^{2} F_{4}^{\prime}}{\partial y \partial t^{\prime}}=\frac{\partial^{2} F_{1}^{\prime}}{\partial y \partial z}=-\frac{\partial^{2} F_{2}}{\partial y^{2}}=-\frac{\partial^{2} F_{3}^{\prime}}{\partial y \partial x} \\
\frac{\partial^{2} F_{4}^{\prime}}{\partial t^{\prime} \partial z}=\frac{\partial^{2} F_{1}^{\prime}}{\partial z^{2}}=-\frac{\partial^{2} F_{2}^{\prime}}{\partial z \partial y}=-\frac{\partial^{2} F_{3}^{\prime}}{\partial z \partial x} .
\end{gathered}
$$

\section{Coupling Equations}

Using the equalities sets in the above relations, one obtains that:

$$
\begin{gathered}
\frac{\partial^{2} \phi}{\partial t^{\prime 2}}-4 \pi G \rho(t, x, y, z)-\frac{\partial^{2} A_{x}}{\partial t^{\prime} \partial x}-\frac{\partial^{2} A_{y}}{\partial t^{\prime} \partial y}+\frac{\partial^{2} A_{z}}{\partial t^{\prime} \partial z}=0 \\
-\frac{1}{c^{2}} \frac{\partial^{2} A_{x}}{\partial t^{2}}\left(t^{\prime}, x, y, z\right)+\frac{4 \pi}{c} J_{x}(t, x, y, z)+\frac{\partial^{2} \phi}{\partial t^{\prime} \partial x}-\frac{\partial^{2} A_{y}}{\partial t^{\prime} \partial z}-\frac{\partial^{2} A_{z}}{\partial t^{\prime} \partial y}=0 \\
-\frac{1}{c^{2}} \frac{\partial^{2} A_{y}}{\partial t^{\prime 2}}(t, x, y, z)+\frac{4 \pi}{c} J_{y}(t, x, y, z)+\frac{\partial^{2} \phi}{\partial t^{\prime} \partial y}-\frac{\partial^{2} A_{x}}{\partial t^{\prime} \partial z}-\frac{\partial^{2} A_{z}}{\partial t^{\prime} \partial x}=0
\end{gathered}
$$

Finally, let us associating the system of equations:

$$
-\frac{1}{c^{2}} \frac{\partial^{2} A_{z}}{\partial t^{\prime}}\left(t^{\prime}, x, y, z\right)+4 \frac{\pi}{c} J_{z}\left(t^{\prime}, x, y, z\right)+\frac{\partial^{2} \phi}{\partial t^{\prime} \partial z}+\frac{\partial^{2} A_{x}}{\partial t^{\prime} \partial y}+\frac{\partial^{2} A_{y}}{\partial t^{\prime} \partial x}=0 .
$$

Adding the equations (32), (33), (34) and (35), has:

$$
\begin{aligned}
\frac{\partial^{2} \phi}{\partial t^{2}}-\frac{1}{c^{2}} & {\left[\frac{\partial^{2} A_{x}}{\partial t^{2}}+\frac{\partial^{2} A_{y}}{\partial t^{2}}+\frac{\partial^{2} A_{z}}{\partial t^{2}}\right]+\frac{4 \pi}{c}\left(J_{x}+J_{y}+J_{z}\right)-4 \pi G \rho } \\
& =\frac{\partial}{\partial t^{\prime}}\left[\frac{\partial}{\partial x}\left(A_{x}-\phi+A_{z}-A_{y}\right)+\frac{\partial}{\partial y}\left(A_{y}+A_{z}-\phi-A_{x}\right)\right.
\end{aligned}
$$




$$
\left.+\frac{\partial}{\partial z}\left(-\phi-A_{z}+A_{y}+A_{x}\right)\right]
$$

Making trivial modifications in equation (36), we have:

$$
\begin{aligned}
& \frac{\partial^{2} \phi}{\partial t^{\prime 2}}-\frac{1}{c^{2}} \frac{\partial}{\partial t^{\prime}}\left\langle\left(\frac{\partial A_{x}}{\partial t^{\prime}}, \frac{\partial A_{y}}{\partial t^{\prime}}, \frac{\partial A_{z}}{\partial t^{\prime}}\right) \cdot(1,1,1)\right\rangle+\frac{4 \pi}{c}\left\langle\left(J_{x}, J_{y}, J_{z}\right),(1,1,1)\right\rangle-4 \pi G \rho \\
&=\frac{\partial}{\partial t^{\prime}}\left[\frac{\partial}{\partial x}\left(A_{x}-\phi+A_{z}-A_{y}\right)+\right. \frac{\partial}{\partial y}\left(A_{y}+A_{z}-\phi-A_{x}\right) \\
&\left.+\frac{\partial}{\partial z}\left(-\phi-A_{z}+A_{y}+A_{x}\right)\right]
\end{aligned}
$$

But

$$
-\frac{\partial \mathbf{A}}{\partial t^{\prime}}=\mathbf{E}+\nabla \varphi
$$

and

$$
-\Upsilon \mathbf{A} \cdot(1,1,1)=\frac{4 \pi}{c} \mathbf{J}
$$

where $\varphi$ and $\Upsilon$ is electrical potential and d'Alembertian operator respectively. Replacing in (37), have

$$
\begin{aligned}
& \frac{\partial^{2} \phi}{\partial t^{\prime 2}}+\frac{1}{c^{2}} \frac{\partial}{\partial t^{\prime}}\langle(\mathbf{E}+\nabla \varphi) \cdot(1,1,1)\rangle-\langle\Upsilon \mathbf{A},(1,1,1)\rangle-4 \pi G \rho \\
= & \frac{\partial}{\partial t^{\prime}}\left[\frac{\partial}{\partial x}\left(A_{x}-\phi+A_{z}-A_{y}\right)+\frac{\partial}{\partial y}\left(A_{y}+A_{z}-\phi-A_{x}\right)+\frac{\partial}{\partial z}\left(-\phi-A_{z}+A_{y}+A_{x}\right)\right]
\end{aligned}
$$

it is also known that

$$
\nabla \cdot \mathbf{B}=0
$$

where $\mathbf{B}$ is magnetic field. So:

$$
\begin{aligned}
& \frac{\partial^{2} \phi}{\partial t^{\prime}}+\nabla \cdot \mathbf{B}+\frac{1}{c^{2}} \frac{\partial}{\partial t^{\prime}}\langle(\mathbf{E}+\nabla \varphi) \cdot(1,1,1)\rangle-\langle\Upsilon \mathbf{A},(1,1,1)\rangle-4 \pi G \rho \\
= & \frac{\partial}{\partial t^{\prime}}\left[\frac{\partial}{\partial x}\left(A_{x}-\phi+A_{z}-A_{y}\right)+\frac{\partial}{\partial y}\left(A_{y}+A_{z}-\phi-A_{x}\right)+\frac{\partial}{\partial z}\left(-\phi-A_{z}+A_{y}+A_{x}\right)\right] .
\end{aligned}
$$

Finally, for values of $t^{\prime}$ small, it has been:

$$
\nabla g=4 \pi G \rho,
$$

where $g$ is gravitational field. Therefore: 


$$
\begin{aligned}
& \frac{\partial^{2} \phi}{\partial t^{\prime}}+\nabla \cdot(\mathbf{B}-g)+\frac{1}{c^{2}} \frac{\partial}{\partial t^{\prime}}\langle(\mathbf{E}+\nabla \varphi) \cdot(1,1,1)\rangle-\langle\Upsilon \mathbf{A},(1,1,1)\rangle \\
= & \frac{\partial}{\partial t^{\prime}}\left[\frac{\partial}{\partial x}\left(A_{x}-\phi+A_{z}-A_{y}\right)+\frac{\partial}{\partial y}\left(A_{y}+A_{z}-\phi-A_{x}\right)+\frac{\partial}{\partial z}\left(-\phi-A_{z}+A_{y}+A_{x}\right)\right] .
\end{aligned}
$$

\section{Concluding Remarks}

The present study showed a single equation involving electrical fields, gravitational and magnetic plus potentials electric, magnetic and gravitational. This is a study that relates these hitherto unrelated fields. The paper concludes:

(i) Potential classics are in the same equation, and there are influences between them;

(ii) The gravitational field was approximated for small values of $t$ for it to be possible to contain the ratio $g$, ie, the only possible relationship between the fields in the classical limit $t^{\prime}$ approaching zero.

\section{References}

[1] J.M. Machado, M.F. Borges, New ramrks on the differenciability of hypercomplex functions, International Journal of Pure and Applied Mathematics, 8, No. 1 (2002), 85-101.

[2] J.A.P.F. Marão, M.F. Borges, Geometrical hypercomplex coupling between electric and gravitational fields, International Journal of Pure and Applied Mathematics, 88, No.4 (2013).

[3] J.A.P.F. Marão, M.F. Borges, A note on the hypercomplex RiemannCauchy like relations for quaternions and laplace equations, International Journal of Pure and Applied Mathematics, 90, No. 4 (2014).

[4] Kunihiko Kodaira, Complex Analysis, Cambridge Studies in Advanced Mathematics; Cambridge University Press Cambridge (2007), 406pp.

[5] J.A.P.F. Marão, M.F. Borges, Geometrical coupling fields of a hypercomplex type, International Journal of Pure and Applied Mathematics, 89, No. 2 (2014). 